Research Paper

\title{
Predictors of the Survival of Primary and Secondary Older Osteosarcoma Patients
}

\author{
Zhan Wang, Bo Wu, Yuanxi Zhou, Xin Huang, Weibo Pan, Meng Liu, Xiaobo Yan, Nong Lin ${ }^{\bowtie}$, Zhaoming \\ $\mathrm{Ye}^{\bowtie}$ \\ Department of Orthopaedics, Centre for Orthopaedic Research, Orthopedics Research Institute of Zhejiang University, The Second Affiliated Hospital, Zhejiang \\ University School of Medicine, 88 Jiefang Road, Hangzhou, Zhejiang 310000, P.R. China \\ $\triangle$ Corresponding authors: Dr. Zhaoming Ye, Department of Orthopaedics, The Second Affiliated Hospital, Zhejiang University School of Medicine, 88 Jiefang \\ Road, Hangzhou, Zhejiang 310000, P.R. China. Tel: +86-13606501549; E-mail: yezhaoming@zju.edu.cn; Dr. Nong Lin, MD; E-mail: linnong@zju.edu.cn \\ (C) The author(s). This is an open access article distributed under the terms of the Creative Commons Attribution License (https://creativecommons.org/licenses/by/4.0/). \\ See http://ivyspring.com/terms for full terms and conditions.
}

Received: 2018.12.28; Accepted: 2019.06.07; Published: 2019.08.07

\begin{abstract}
Purpose: Older osteosarcoma patients have a very poor prognosis and treatment for them remains a challenge. The outcomes and potential prognostic factors of primary or secondary older osteosarcoma patients are rarely documented. Therefore, we examined the prognosis of the two special cohorts to identify possible prognostic factors, and provide optimal treatment strategy for them.

Methods: The Surveillance, Epidemiology, and End Results (SEER) program database was used to identify osteosarcoma patients aged over 40 years from 1973 to 2015 . The prognostic analysis was performed using the Kaplan-Meier method and a Cox proportional hazards regression model.

Results: In total, 1162 primary older osteosarcoma patients and 444 secondary older osteosarcoma patients were eligible for this study. The OS and CSS rates of the primary older osteosarcoma patients at 5-year were $38.5 \%$ and $37.1 \%$, respectively. The 3 - and 5-year OS rates of the secondary older osteosarcoma patients were $22.8 \%$ and $14.6 \%$, respectively. On multivariate analysis of the primary older osteosarcoma patients, age $>60$, male, axial site, high grade, metastasis, tumor size $>10 \mathrm{~cm}$, no surgery, and radiation treatment were negatively associated with OS. In terms of CSS, age, gender, decade of diagnosis, tumor site, tumor grade, tumor stage, tumor size, and surgery were independent prognostic factors. A multivariate Cox regression model showed that secondary older osteosarcoma patients of high grade, metastasis, tumor size $>10 \mathrm{~cm}$, no surgery, and no chemotherapy were independent predictors of decreased OS.

Conclusions: Surgery in combination with chemotherapy should be recommended for the treatment of the secondary older osteosarcoma patients, while for the primary older osteosarcoma patients, only surgery should be recommended.
\end{abstract}

Key words: primary osteosarcoma; secondary osteosarcoma; adults; prognostic factor; therapy

\section{Introduction}

Osteosarcoma is the most common type of primary bone sarcomas and occurs predominantly in children and young adults [1]. The demographic, prognostic, and outcome data of osteosarcoma in children and young adults, or patients of all ages are well documented [2-4]. Current standard treatment of younger osteosarcoma patients includes preoperative chemotherapy, surgical resection of all tumor and postoperative chemotherapy [5]. The 10-year cancer specific survival (CSS) rate for patients with localized/regional osteosarcoma is approximately $70 \%$, while for patients with metastatic disease is $24 \%$ [3]. Age at diagnosis, tumor size and site, pathological fractures, tumor grade, metastasis at presentation, and systemic chemotherapy are all associated with the prognosis of osteosarcoma $[2,3,6,7]$. 
The second incidence peak of primary osteosarcoma is in the older. Coincidentally, secondary osteosarcoma also frequently occurs in the older persons mainly due to Paget's disease or post-radiation and usually has a much poorer outcome compared to primary osteosarcoma [8,9]. Older osteosarcoma patients often have a worse prognosis compared with younger patients [7, 10]. Recently, Iwata et al. [10] reported poor prognoses in 86 elderly osteosarcoma patients, with a 5-year overall survival rate $38.8 \%$. They found that tumor site, metastasis at diagnosis, surgery, and surgical margins were significantly associated with survival, whereas chemotherapy had no influence on survival. However, some studies reported that aggressive treatment with chemotherapy can offer a favorable outcome for older osteosarcoma patients [11-13]. Although osteosarcoma is radioresistant, some studies have reported that radiotherapy is an effect adjuvant treatment for local control [14, 15]. Older osteosarcoma patients may receive radiotherapy due to its lower toxicity compared with systemic chemotherapy $[13,15]$. The effects of radiotherapy on survival of older osteosarcoma patients are rarely studied. Therefore, this study also assessed the prognostic utility of radiotherapy in older osteosarcoma patients.

Optimal treatment for older osteosarcoma patients is still controversial. To obtain deeper insight into primary and secondary older osteosarcoma patients, we analyzed osteosarcoma patients aged over 40 from 1973 to 2015 in the Surveillance, Epidemiology, and End Results (SEER) program database of the National Cancer Institute. This was a large-scale study of older osteosarcoma patients that aimed to confirm the predictors of survival.

\section{Materials and Methods}

\section{Patient population}

A total of 6224 patients diagnosed with osteosarcoma of bone were identified from 1973 to 2015. All patient data were obtained using the case-listing session procedure from the SEER program database. The database is publicly available and does not include unique patient identifiers. This study followed standard guidelines and was approved by the Ethics Committee of the Second Affiliated Hospital of Zhejiang University School of Medicine.

First, the International Classification of Diseases for Oncology, $3^{\text {rd }}$ edition (ICD-O-3) was used to identify patients with osteosarcoma of bone (ICD-O-3 histologic type: 9180-9187, 9192-9195; ICD-O-3 site code: C40.0-40.3, C40.8-41.4, C41.8-41.9), using the case-listing procedure. Only patients aged over 40 were enrolled, by reference to the age at diagnosis. Previous researches also recognized osteosarcoma patients aged over 40 as older osteosarcoma patients. All patients were confirmed histologically, based either on biopsy results or the surgical specimen. One hundred and forty-four patients diagnosed only on the basis of the clinical presentation, or according to the radiography were excluded. Twenty-five patients with unknown therapy were excluded. Sixty-five patients with missing survival information or survival month $\leq 1$ were also excluded. The sequence number was used to classify older patients as having a primary osteosarcoma without any other primary malignant tumors (sequence number $\leq 1)(n=1162)$ and as having osteosarcoma as a secondary malignancy (sequence number $\geq 2)(n=444)$ (Figure. 1). Data extracted from the SEER database included age, gender, race, year of diagnosis, location, tumor grade, tumor stage, tumor type, tumor size, surgical treatment, radiation treatment, chemotherapy, cause of death, and survival time. Surgery or radiation treatment for tumors in our study refers to treatment for local primary tumors. We divided the location into three categories: (1) appendicular (long and short bones of the upper and lower extremities), (2) axial (pelvis and spine), and (3) other locations (mandible, skull, rib, sternum, clavicle, and other atypical locations). Given that age is an important predictor for survival of osteosarcoma, we further divided older osteosarcoma patients into two categories ( $\leq 60$ group and $>60$ group) according to the mean and median age at diagnosis.

\section{Statistical methods}

The statistical analyses were performed using Microsoft Excel 2016 (Microsoft Corp., Redmond, WA, USA) and SPSS software (ver. 21.0; SPSS Inc., Chicago, IL, USA). Overall survival (OS) was defined as the time from diagnosis to death from any cause and cancer-specific survival (CSS) was regarded as the time from diagnosis to death due specifically to cancer. The Kaplan-Meier method was used to draw the OS and CSS curves and calculate median survival. Observations were censored if the patient was alive at the time of the last follow-up. Univariate analyses were performed using the Kaplan-Meier method with the log-rank test. Multivariate analysis was used to determine the independent predictors of OS and CSS with a Cox proportional hazards regression model. The hazard ratios (HRs) and corresponding 95\% confidence intervals (CIs) were used to show the effect of various factors on OS and CSS. Differences were deemed statistically significant if the P-value was less than 0.05 . 


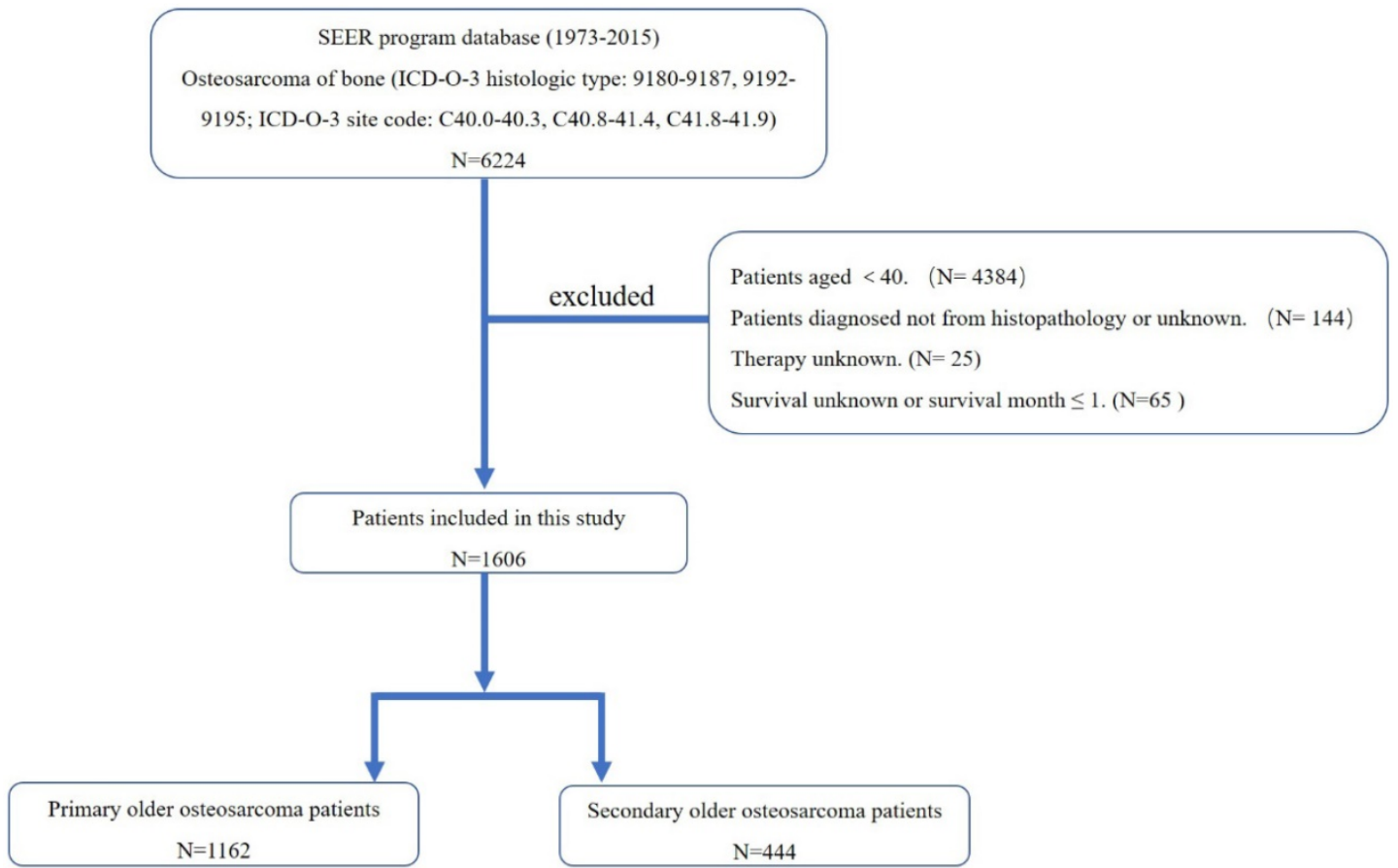

Figure 1. The flow chart for selection of study population. (Abbreviations: SEER, Surveillance, Epidemiology, and End Results; ICD-O-3, international classification of diseases for oncology, 3rd edition.)

\section{Results}

\section{Demographic and clinical characteristics of osteosarcoma patients aged over $\mathbf{4 0}$}

In total, 1606 patients were eligible for our study, including 1162 primary older osteosarcoma patients and 444 secondary older osteosarcoma patients. In primary older osteosarcoma cohort, the mean and median patient age at diagnosis were 58 and 56 years, respectively. In terms of location, $54.8 \%$ tumors were located in the extremities, $19.4 \%$ in the axial skeleton, and $25.8 \%$ in other sites. Histologically, $13.6 \%$ of the cases were low grade, and $52.7 \%$ were high grade. The majority of the patients were diagnosed as osteosarcoma, not otherwise specified (NOS) (71.9\%). Information on the tumor size was available in $62.3 \%$ cases, and was categorized into three groups. More than two thirds of the patients $(75.5 \%)$ received local surgery, 272 patients $(23.4 \%)$ received radiation treatment, and about half of patients had chemotherapy. Ultimately, 781 patients $(67.2 \%)$ died, of whom 631 died of cancer. The 3- and 5-year OS rates of the entire cohort were $47.1 \%$ and $38.5 \%$, respectively. The 3- and 5-year CSS rates were $45.6 \%$ and $37.1 \%$, respectively (Table 1 ). The median OS and CSS were $30.0 \pm 2.6$ and $28.0 \pm 2.4$ months, respectively, suggesting a poor prognosis for this cohort (Table 2).

In secondary older osteosarcoma cohort, the mean and median patient age at diagnosis were 66 and 67 years, respectively. Compared with the primary older osteosarcoma, secondary older osteosarcoma shows a predilection for axial sites $(33.8 \%)$. Histologically, $6.8 \%$ of the cases were low grade, and $59.0 \%$ were high grade. The majority of the cases were also osteosarcoma, not otherwise specified (NOS) $(65.5 \%)$. Information on the tumor size was available in $53.2 \%$ cases, and was categorized into three groups. More than half of the patients $(64.9 \%)$ received local surgery, 104 patients $(23.4 \%)$ received radiation treatment, and about half of patients had chemotherapy. The 3- and 5-year OS rates of this cohort were $22.8 \%$ and $14.6 \%$, respectively (Table 1 ). The median OS of this population was $12.0 \pm 0.9$, suggesting a poorer prognosis than primary older osteosarcoma patients (Table 2).

\section{Univariate analyses of variables associated with OS or CSS in osteosarcoma patients aged over 40}

Univariate analyses of variables associated with survival of older osteosarcoma patients are shown in Table 3. Among primary older osteosarcoma patients, univariate analyses revealed that race was not associated with either OS or CSS. Female patients had significantly better outcomes than male patients, with a longer median survival. OS and CSS showed a statistically significant difference in survival based on decade of diagnosis. Patients with axial tumor location had worse outcomes than those with appendicular or other tumor locations. Both OS and CSS differed significantly with tumor grade, with a high tumor grade portending a worse prognosis. 
Table 1. Demographic and clinical characteristics of primary osteosarcoma patients $(n=1162)$ and secondary osteosarcoma patients $(n=444)$ aged over 40 identified in the SEER database from 1973 to 2015

\begin{tabular}{|c|c|c|}
\hline Variable & Primary & Secondary \\
\hline \multicolumn{3}{|l|}{ Age(years) } \\
\hline Mean & 58 & 66 \\
\hline Median & 56 & 67 \\
\hline$\leq 60$ & $710(61.1 \%)$ & $155(34.9 \%)$ \\
\hline$>60$ & $452(38.9 \%)$ & $289(65.1 \%)$ \\
\hline \multicolumn{3}{|l|}{ Gender } \\
\hline Female & $533(45.9 \%)$ & $221(49.8 \%)$ \\
\hline Male & $629(54.1 \%)$ & $223(50.2 \%)$ \\
\hline \multicolumn{3}{|l|}{ Decade of diagnosis } \\
\hline $1970 \mathrm{~s}$ & $83(7.1 \%)$ & $21(4.7 \%)$ \\
\hline $1980 \mathrm{~s}$ & $130(11.2 \%)$ & $37(8.3 \%)$ \\
\hline $1990 \mathrm{~s}$ & $187(16.1 \%)$ & $93(20.9 \%)$ \\
\hline$\geq 2000 \mathrm{~s}^{\mathrm{a}}$ & $762(65.6 \%)$ & $293(66.0 \%)$ \\
\hline \multicolumn{3}{|l|}{ Race } \\
\hline White & $914(78.7 \%)$ & $362(81.5 \%)$ \\
\hline Black & $157(13.5 \%)$ & $51(11.5 \%)$ \\
\hline Other & $91(7.8 \%)$ & $31(7.0 \%)$ \\
\hline \multicolumn{3}{|l|}{ Location } \\
\hline Appendicular & $637(54.8 \%)$ & $149(33.6 \%)$ \\
\hline Axial & $225(19.4 \%)$ & $150(33.8 \%)$ \\
\hline Other locations & $300(25.8 \%)$ & $145(32.7 \%)$ \\
\hline \multicolumn{3}{|l|}{ Tumor type } \\
\hline Osteosarcoma, not otherwise specified (NOS) & $836(71.9 \%)$ & $291(65.5 \%)$ \\
\hline Chondroblastic osteosarcoma & $114(9.8 \%)$ & $44(9.9 \%)$ \\
\hline Fibroblastic osteosarcoma & $89(7.7 \%)$ & $24(5.4 \%)$ \\
\hline Parosteal osteosarcoma & $55(4.7 \%)$ & $3(0.7 \%)$ \\
\hline Telangiectatic osteosarcoma & $24(2.1 \%)$ & $3(0.7 \%)$ \\
\hline Central osteosarcoma & $17(1.5 \%)$ & $6(1.4 \%)$ \\
\hline Small cell osteosarcoma & $11(0.9 \%)$ & $1(0.2 \%)$ \\
\hline Periosteal osteosarcoma & $11(0.9 \%)$ & - \\
\hline Intraosseous well differentiated osteosarcoma & $3(0.3 \%)$ & $1(0.2 \%)$ \\
\hline High grade surface osteosarcoma & $2(0.2 \%)$ & $1(0.2 \%)$ \\
\hline Osteosarcoma in Paget disease of bone & - & $70(15.8 \%)$ \\
\hline \multicolumn{3}{|l|}{ Tumor gradeb } \\
\hline Low & $158(13.6 \%)$ & $30(6.8 \%)$ \\
\hline High & $612(52.7 \%)$ & $262(59.0 \%)$ \\
\hline Unknown & $392(33.7 \%)$ & $152(34.2 \%)$ \\
\hline \multicolumn{3}{|l|}{ Tumor stage } \\
\hline Localized & $354(30.5 \%)$ & $115(25.9 \%)$ \\
\hline Regional & $456(39.2 \%)$ & $178(40.1 \%)$ \\
\hline Distant & $264(22.7 \%)$ & $106(23.9 \%)$ \\
\hline Unknown & $88(7.6 \%)$ & $45(10.1 \%)$ \\
\hline \multicolumn{3}{|l|}{ Tumor size } \\
\hline$<5 \mathrm{~cm}$ & $169(14.5 \%)$ & $66(14.9 \%)$ \\
\hline $5-10 \mathrm{~cm}$ & $316(27.2 \%)$ & $96(21.6 \%)$ \\
\hline$>10 \mathrm{~cm}$ & $239(20.6 \%)$ & $74(16.7 \%)$ \\
\hline Unknown & $438(37.7 \%)$ & $208(46.8 \%)$ \\
\hline \multicolumn{3}{|l|}{ Surgery } \\
\hline Yes & $877(75.5 \%)$ & $288(64.9 \%)$ \\
\hline No & $285(24.5 \%)$ & $156(35.1 \%)$ \\
\hline \multicolumn{3}{|l|}{ Radiation treatment } \\
\hline Yes & $272(23.4 \%)$ & $104(23.4 \%)$ \\
\hline No & $890(76.6 \%)$ & $340(76.6 \%)$ \\
\hline \multicolumn{3}{|l|}{ Chemotherapy } \\
\hline Yes & $603(51.9 \%)$ & $221(49.8 \%)$ \\
\hline No & $559(48.1 \%)$ & $223(50.2 \%)$ \\
\hline \multicolumn{3}{|l|}{ Dead } \\
\hline Yes & $781(67.2 \%)$ & $376(84.7 \%)$ \\
\hline No & $381(32.8 \%)$ & $68(15.3 \%)$ \\
\hline 3-year OS rate & $47.1 \%$ & $22.8 \%$ \\
\hline 3-year CSS rate & $45.6 \%$ & - \\
\hline 5-year OS rate & $38.5 \%$ & $14.6 \%$ \\
\hline 5-year CSS rate & $37.1 \%$ & - \\
\hline
\end{tabular}

a: 2000-2015 year; b: Low: ICD-O-3 Grade 1 (well differentiated) and Grade 2 (moderately differentiated); High: ICD-O-3 Grade 3 (poorly differentiated) and Grade 4 (undifferentiated anaplastic).

Tumor stage was associated with significant differences in OS and CSS, with metastasis predicting a worse prognosis. Similarly, tumor size was associated with significant differences in OS and CSS, with smaller tumor size predicting a better prognosis. In terms of treatment, patients who underwent surgical treatment had better OS and CSS than those who did not (Fig.2A and 2D). However, patients who received radiation treatment had worse OS and CSS than those who did not (Fig.2B and 2E). No significant difference in OS or CSS, based on chemotherapy was observed (Fig.2C and 2F).

Among secondary older osteosarcoma patients, only age, tumor site, tumor grade, tumor stage, tumor size, surgery (Fig.3A), radiation treatment (Fig.3B) and chemotherapy (Fig.3C) showed significant differences in OS.

\section{Multivariate analysis of independent predictors of OS or CSS in osteosarcoma patients aged over 40}

The prognostic factors for older osteosarcoma patients are shown in Table 4. On multivariate analysis of all primary older osteosarcoma patients, age $>60$, male, axial site, high grade, metastasis, tumor size $>10 \mathrm{~cm}$, no surgery, and radiation treatment were negatively associated with OS. In terms of CSS, age, gender, decade of diagnosis, tumor site, tumor grade, tumor stage, tumor size, and surgery were independent prognostic factors. A multivariate Cox regression model showed that secondary older osteosarcoma patients of high grade, metastasis, tumor size $>10 \mathrm{~cm}$, no surgery, and no chemotherapy were correlated with higher risk of mortality.

\section{Discussion}

Osteosarcoma is the most frequent primary malignant bone tumor, and occurs predominantly in children and young adults [16, 17]. Recently, the occurrence of older osteosarcoma patients has increased, and the prognosis of them is very poor [10]. Because cases of osteosarcoma patients aged over 40 are rare, few studies have documented the prognostic factors of this special cohort [10]. This is the largest study to report outcomes of older osteosarcoma patients, and the first to analyze the prognosis of primary and secondary osteosarcoma patients at the same time. The strengths of our study were its size (1162 primary older osteosarcoma patients and 444 secondary older osteosarcoma patients). We also analyzed the effects of controversial risk factor- 
radiotherapy on survival of older osteosarcoma patients.

Patients with osteosarcoma aged over 40 exhibit different clinical characteristics compared with children and young adults [18]. Previous studies found that metastasis at presentation was more frequent in older patients than younger patients. Nearly a quarter of the patients in our study presented metastatic disease at diagnosis, which was higher than other studies $[13,19,20]$ but similar with one previous report [10]. Axial osteosarcoma occurred more frequently in the older than adolescents. Our study revealed that the incidence of axial osteosarcoma in primary older osteosarcoma patients was $19.4 \%$, which was lower than that of previous studies [20, 21]. Compared with the primary older osteosarcoma, secondary older osteosarcoma showed a predilection for axial sites (33.8\%). Additionally, in our study, osteosarcoma, NOS was the most common histological type in both primary and secondary older osteosarcoma, which were similar to a previous study of all osteosarcoma [3].

Due to the frequent axial occurrence and metastasis in this age group, the outcome is usually poor. Iwata et al. [10] found that the 5-year OS and Event-free survival (EFS) in 86 osteosarcoma patients aged over 40 were $38 \%$ and $34 \%$, respectively. Carsi et al. [21] reported that the 5-year OS and EFS rates were $41.64 \%$ and $32.54 \%$, respectively. However, Ozkurt et al. [11] reported that the survival rate of the same age group was $76.2 \%$ at 2 years and $72.8 \%$ at 5 years in 36 cases. It is possible that their follow-up time is too short (median: 7 months) and treatment is more aggressive.

Table 2. Median survival data (month) of osteosarcoma patients aged over 40

\begin{tabular}{|c|c|c|c|c|c|c|}
\hline Variable & Primary & & & & Secondary & \\
\hline & $\begin{array}{l}\text { OS } \\
\text { (estimate } \pm S E)\end{array}$ & $95 \% \mathrm{CI}$ & CSS (estimate \pm SE ) & $95 \% \mathrm{CI}$ & $\begin{array}{l}\text { OS } \\
\text { (estimate } \pm S E)\end{array}$ & $95 \% \mathrm{CI}$ \\
\hline Overall & $30.0 \pm 2.6$ & $24.8-35.2$ & $28.0 \pm 2.4$ & $23.4-32.6$ & $12.0 \pm 0.9$ & $10.3-13.7$ \\
\hline \multicolumn{7}{|l|}{ Age (years) } \\
\hline$\leq 60$ & $56.0 \pm 7.0$ & $42.2-69.8$ & $53.0 \pm 7.3$ & $38.7-67.3$ & $20.0 \pm 2.5$ & $15.0-25.0$ \\
\hline$>60$ & $14.0 \pm 1.3$ & $11.5-16.5$ & $12.0 \pm 1.0$ & $10.0-14.0$ & $9.0 \pm 0.9$ & 7.3-10.7 \\
\hline \multicolumn{7}{|l|}{ Gender } \\
\hline Female & $36.0 \pm 5.3$ & $25.6-46.4$ & $35.0 \pm 4.9$ & $25.5-44.5$ & $11.0 \pm 1.4$ & $8.3-13.7$ \\
\hline Male & $27.0 \pm 3.2$ & $20.7-33.3$ & $25.0 \pm 2.4$ & 20.3-29.7 & $12.0 \pm 1.3$ & $9.4-14.6$ \\
\hline \multicolumn{7}{|c|}{ Decade of diagnosis } \\
\hline $1970 \mathrm{~s}$ & $14.0 \pm 2.9$ & $8.3-19.7$ & $10.0 \pm 1.9$ & $6.2-13.8$ & $11.0 \pm 2.8$ & $5.4-16.6$ \\
\hline $1980 \mathrm{~s}$ & $18.0 \pm 2.9$ & $12.4-23.6$ & $14.0 \pm 2.7$ & $8.7-19.3$ & $9.0 \pm 1.5$ & $6.1-11.9$ \\
\hline $1990 \mathrm{~s}$ & $36.0 \pm 8.8$ & $18.7-53.3$ & $23.0 \pm 6.0$ & $11.3-34.7$ & $17.0 \pm 4.0$ & $9.1-24.9$ \\
\hline$\geq 2000 \mathrm{~s}^{\mathrm{a}}$ & $35.0 \pm 3.7$ & $27.7-42.3$ & $37.0 \pm 4.1$ & $28.9-45.1$ & $12.0 \pm 0.8$ & $10.5-13.5$ \\
\hline \multicolumn{7}{|l|}{ Race } \\
\hline White & $28.0 \pm 2.8$ & $22.5-33.5$ & $26.0 \pm 2.7$ & $20.8-31.2$ & $12.0 \pm 1.1$ & $9.8-14.2$ \\
\hline Black & $37.0 \pm 8.0$ & $21.3-52.7$ & $29.0 \pm 7.0$ & $15.3-42.7$ & $12.0 \pm 2.2$ & $7.7-16.3$ \\
\hline Other & $37.0 \pm 10.3$ & $16.8-57.2$ & $39.0 \pm 11.1$ & $17.2-60.8$ & $10.0 \pm 1.0$ & $8.0-12.0$ \\
\hline \multicolumn{7}{|l|}{ Location } \\
\hline Appendicular & $45.0 \pm 4.9$ & $35.4-54.6$ & $39.0 \pm 4.5$ & $30.2-47.8$ & $18.0 \pm 2.7$ & $12.7-23.3$ \\
\hline Axial & $12.0 \pm 1.4$ & $9.2-14.8$ & $12.0 \pm 1.5$ & $9.1-14.9$ & $8.0 \pm 0.8$ & $6.3-9.7$ \\
\hline Other locations & $40.0 \pm 8.3$ & $23.7-56.3$ & $38.0 \pm 7.9$ & $22.6-53.4$ & $13.0 \pm 1.0$ & $11.0-15.0$ \\
\hline \multicolumn{7}{|l|}{ Tumor gradeb } \\
\hline Low & $191.0 \pm 22.6$ & $146.8-235.3$ & NA & NA & $41.0 \pm 40.2$ & $0.000-119.7$ \\
\hline High & $27.0 \pm 2.8$ & $21.5-32.5$ & $26.0 \pm 2.8$ & $20.5-31.5$ & $13.0 \pm 1.1$ & $10.8-15.2$ \\
\hline \multicolumn{7}{|l|}{ Tumor stage } \\
\hline Localized & $124.0 \pm 26.7$ & $71.6-176.4$ & $241.0 \pm 74.5$ & $94.9-387.1$ & $19.0 \pm 3.3$ & $12.5-25.5$ \\
\hline Regional & $42.0 \pm 6.0$ & $30.2-53.8$ & $37.0 \pm 5.0$ & $27.1-46.9$ & $16.0 \pm 1.7$ & $12.7-19.3$ \\
\hline Distant & $7.0 \pm 0.7$ & $5.6-8.4$ & $7.0 \pm 0.8$ & $5.4-8.6$ & $6.0 \pm 0.8$ & $4.5-7.5$ \\
\hline \multicolumn{7}{|l|}{ Tumor size } \\
\hline$<5 \mathrm{~cm}$ & $160.0 \pm 37.6$ & $86.4-233.6$ & NA & NA & $22.0 \pm 2.7$ & $16.6-27.4$ \\
\hline $5-10 \mathrm{~cm}$ & $52.0 \pm 9.2$ & $34.0-70.0$ & $52.0 \pm 10.3$ & $31.8-72.2$ & $19.0 \pm 2.5$ & $14.2-23.8$ \\
\hline$>10 \mathrm{~cm}$ & $19.0 \pm 1.6$ & $15.8-22.2$ & $19.0 \pm 1.8$ & $15.4-22.6$ & $10.0 \pm 1.2$ & $7.6-12.4$ \\
\hline \multicolumn{7}{|l|}{ Surgery } \\
\hline Yes & $50.0 \pm 5.7$ & $38.7-61.3$ & $45.0 \pm 4.7$ & $35.8-54.2$ & $18.0 \pm 1.7$ & $14.7-21.3$ \\
\hline No & $8.0 \pm 1.0$ & $6.1-9.9$ & $9.0 \pm 1.0$ & $7.0-11.0$ & $7.0 \pm 0.6$ & $5.8-8.2$ \\
\hline \multicolumn{7}{|c|}{ Radiation treatment } \\
\hline Yes & $14.0 \pm 1.2$ & $11.6-16.4$ & $14.0 \pm 1.4$ & $11.2-16.8$ & $8.0 \pm 1.2$ & $5.6-10.4$ \\
\hline No & $46.0 \pm 4.7$ & $36.8-55.2$ & $41.0 \pm 4.7$ & $31.7-50.3$ & $12.0 \pm 1.0$ & $10.0-14.0$ \\
\hline \multicolumn{7}{|l|}{ Chemotherapy } \\
\hline Yes & $29.0 \pm 2.9$ & $23.4-34.6$ & $28.0 \pm 2.8$ & $22.6-33.4$ & $15.0 \pm 1.1$ & $12.9-17.1$ \\
\hline No & $30.0 \pm 5.6$ & $19.1-40.9$ & $26.0 \pm 4.4$ & $17.5-34.6$ & $9.0 \pm 1.2$ & $6.6-11.4$ \\
\hline
\end{tabular}

OS: overall survival, CSS: cancer-specific survival, N/A means that the median survival time was not available due to death event occurring in fewer than $50 \%$ of cases in the cohort. SE: standard error. 
Table 3. Univariate analyses of variables in osteosarcoma patients aged over 40 using Kaplan-Meier method

\begin{tabular}{|c|c|c|c|}
\hline \multirow[t]{2}{*}{ Variable } & \multicolumn{2}{|l|}{ Primary } & \multirow{2}{*}{$\begin{array}{l}\text { Secondary } \\
\text { OS (Log-rank p } \\
\text { value) }\end{array}$} \\
\hline & $\begin{array}{l}\text { OS (Log-rank p } \\
\text { value) }\end{array}$ & $\begin{array}{l}\text { CSS (Log-rank p } \\
\text { value) }\end{array}$ & \\
\hline Age $(\leq 60 \mathrm{vs}>60)$ & $<0.001$ & $<0.001$ & $<0.001$ \\
\hline Gender & 0.013 & 0.007 & 0.750 \\
\hline Decade of diagnosis & $<0.001$ & $<0.001$ & 0.200 \\
\hline$\geq 2000$ sa vs1970s & $<0.001$ & $<0.001$ & - \\
\hline$\geq 2000$ sa vs 1980 s & 0.002 & $<0.001$ & - \\
\hline$\geq 2000$ sa vs 1990 s & 0.791 & 0.058 & - \\
\hline 1990s vs 1970s & 0.006 & $<0.001$ & - \\
\hline 1990 s vs 1980 s & 0.050 & 0.004 & - \\
\hline 1980 s vs 1970 s & 0.224 & 0.004 & - \\
\hline Race & 0.757 & 0.430 & 0.396 \\
\hline Location & $<0.001$ & $<0.001$ & $<0.001$ \\
\hline Axial vs Appendicular & $<0.001$ & $<0.001$ & $<0.001$ \\
\hline $\begin{array}{l}\text { Axial vs Other } \\
\text { locations }\end{array}$ & $<0.001$ & $<0.001$ & 0.001 \\
\hline $\begin{array}{l}\text { Appendicular vs Other } \\
\text { locations }\end{array}$ & 0.938 & 0.861 & 0.408 \\
\hline $\begin{array}{l}\text { Tumor gradeb (Low vs } \\
\text { High) }\end{array}$ & $<0.001$ & $<0.001$ & 0.001 \\
\hline Tumor stage & $<0.001$ & $<0.001$ & $<0.001$ \\
\hline Distant vs Localized & $<0.001$ & $<0.001$ & $<0.001$ \\
\hline Distant vs Regional & $<0.001$ & $<0.001$ & $<0.001$ \\
\hline Regional vs Localized & $<0.001$ & $<0.001$ & 0.252 \\
\hline Tumor size & $<0.001$ & $<0.001$ & $<0.001$ \\
\hline$>10 \mathrm{~cm}$ vs $<5 \mathrm{~cm}$ & $<0.001$ & $<0.001$ & $<0.001$ \\
\hline$>10 \mathrm{~cm}$ vs $5-10 \mathrm{~cm}$ & $<0.001$ & $<0.001$ & 0.001 \\
\hline $5-10 \mathrm{~cm}$ vs $<5 \mathrm{~cm}$ & 0.001 & 0.003 & 0.235 \\
\hline Surgery & $<0.001$ & $<0.001$ & $<0.001$ \\
\hline Radiation treatment & $<0.001$ & $<0.001$ & 0.022 \\
\hline Chemotherapy & 0.581 & 0.742 & 0.002 \\
\hline
\end{tabular}

Ferrari et al. [12] reported that the 5-year OS of bone-sarcoma patents aged over 40 and synchronous metastases was $22 \%$. In our study, the 3- and 5-year OS rates of the primary older osteosarcoma were $47.1 \%$ and $38.5 \%$, respectively, while the 3 - and 5 -year OS rates of the secondary older osteosarcoma were $22.8 \%$ and $14.6 \%$, suggesting a pretty poor outcome. Thus, it is necessary to explore prognostic factors to better guide the management of such patients.

Age younger than 60 years was considered as a prognostic factor for improved survival for primary older osteosarcoma but not for secondary older osteosarcoma. Male sex was identified as an independent risk factor for decreased OS and CSS. But for secondary older osteosarcoma, gender was not associated with OS. Many studies also identified male sex as a poor prognostic value for osteosarcoma $[3,22$, 23]. Frequent aggressive tumors or poorer response to treatment in males may account for it [3]. An appendicular location of the osteosarcoma was associated with a better outcome compared with an axial location, which was similar with other reports $[2$, $3,10]$. Tumor grade and stage were generally recognized as a very important predictor of osteosarcoma $[3,10]$.
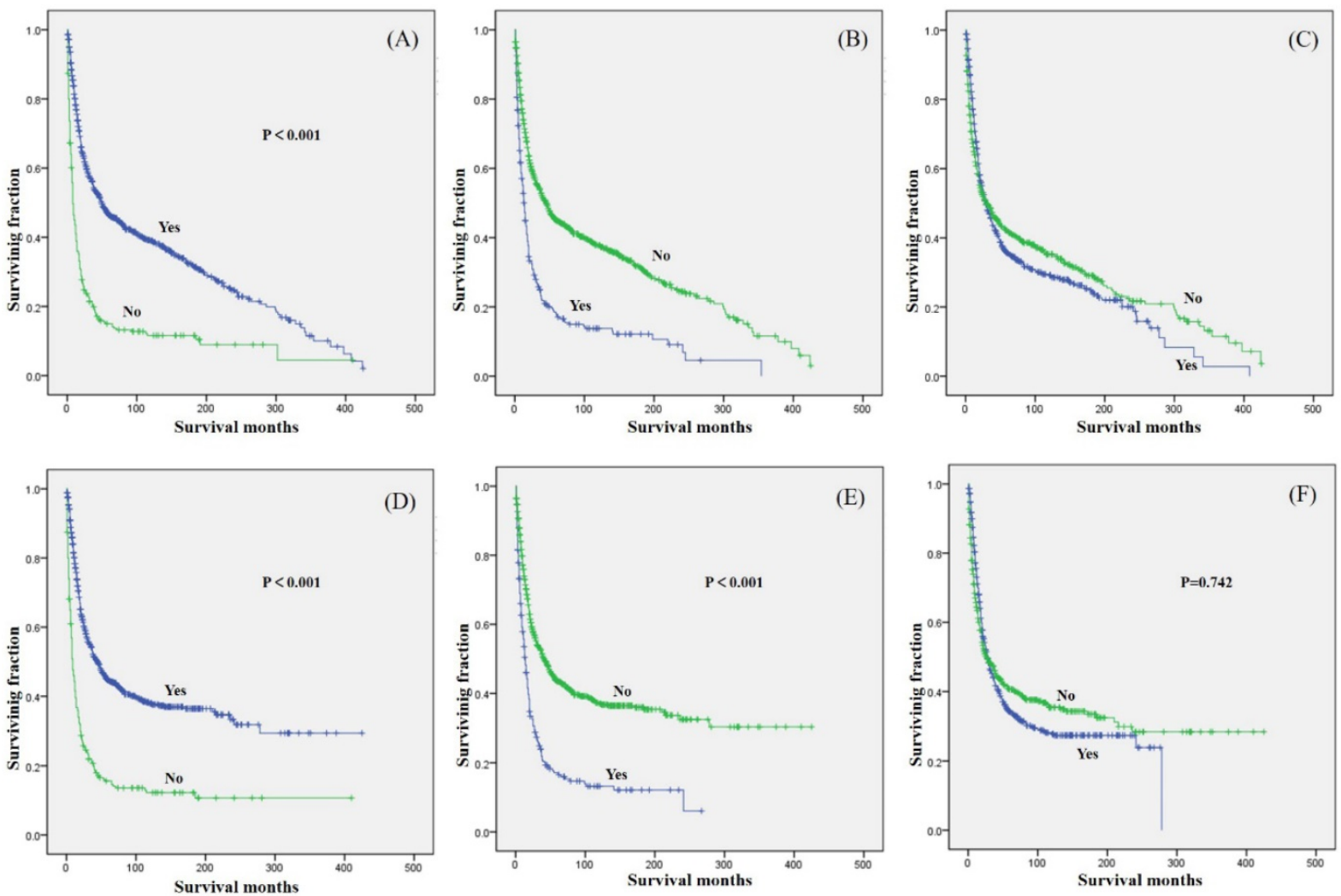

Figure 2. Kaplan-Meier method estimated OS and CSS in primary osteosarcoma patients aged over 40 years. OS stratified by (A) surgery, (B) radiation, and (C) chemotherapy. CSS stratified by (D) surgery, (E) radiation, and (F) chemotherapy. 

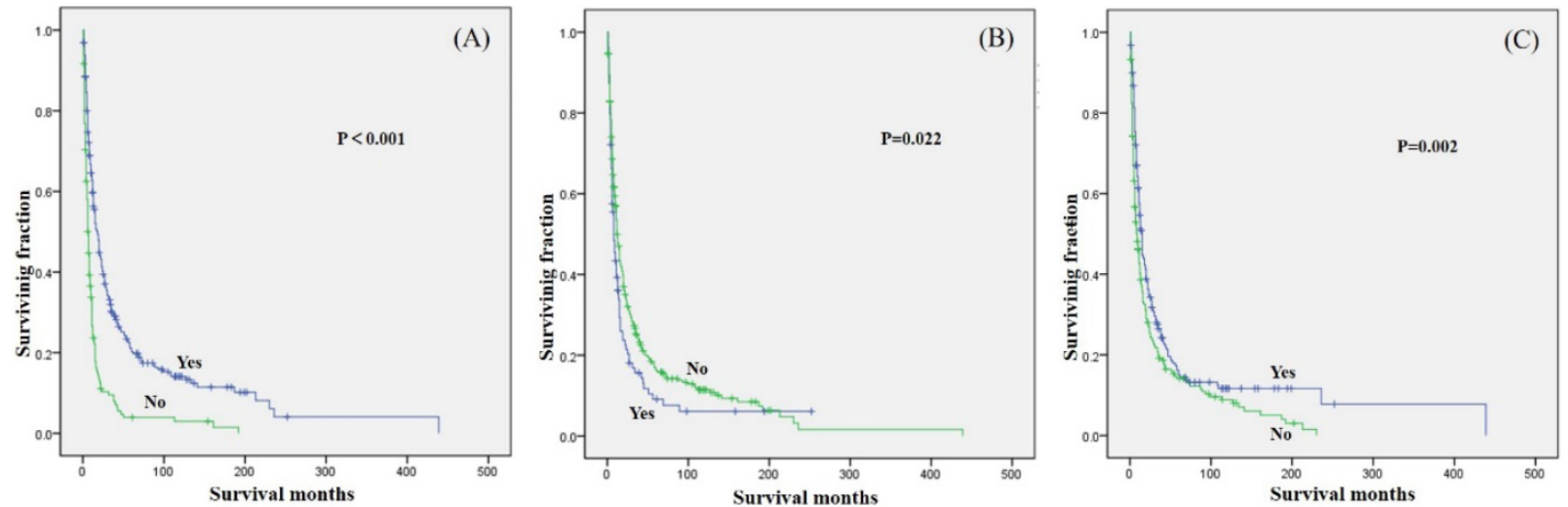

Figure 3. Kaplan-Meier method estimated OS in secondary osteosarcoma patients aged over 40 years stratified by (A) surgery, (B) radiation, and (C) chemotherapy.

Table 4. Multivariate analyses for OS and CSS for osteosarcoma patients aged over 40

\begin{tabular}{|c|c|c|c|c|c|c|}
\hline \multirow[t]{3}{*}{ Variable } & \multicolumn{4}{|l|}{ Primary } & \multicolumn{2}{|l|}{ Secondary } \\
\hline & \multicolumn{2}{|l|}{ OS } & \multicolumn{2}{|l|}{ CSS } & \multicolumn{2}{|l|}{ OS } \\
\hline & Hazard Ratio(95\% CI) & P value & Hazard Ratio(95\% CI) & $P$ value & Hazard Ratio(95\% CI) & $P$ value \\
\hline \multicolumn{7}{|l|}{ Age (years) } \\
\hline$\leq 60$ & 1 & & 1 & & 1 & \\
\hline$>60$ & $1.887(1.624-2.194)$ & $<0.001$ & $1.927(1.622-2.288)$ & $<0.001$ & $1.251(0.981-1.596)$ & 0.071 \\
\hline \multicolumn{7}{|l|}{ Gender } \\
\hline Female & 1 & & 1 & & - & \\
\hline Male & $1.185(1.023-1.373)$ & 0.024 & $1.181(1.002-1.392)$ & 0.047 & - & - \\
\hline \multicolumn{7}{|c|}{ Decade of diagnosis } \\
\hline 1970 s & 1 & & 1 & & - & \\
\hline 1980 s & $1.099(0.819-1.476)$ & 0.528 & $0.855(0.607-1.206)$ & 0.373 & - & - \\
\hline 1990s & $0.870(0.649-1.168)$ & 0.354 & $0.642(0.456-0.903)$ & 0.011 & - & - \\
\hline$\geq 2000 s^{a}$ & $0.804(0.612-1.057)$ & 0.119 & $0.526(0.387-0.715)$ & $<0.001$ & - & - \\
\hline \multicolumn{7}{|l|}{ Location } \\
\hline Appendicular & 1 & & 1 & & 1 & \\
\hline Axial & $1.708(1.418-2.057)$ & $<0.001$ & $1.748(1.427-2.143)$ & $<0.001$ & $1.280(0.979-1.673)$ & 0.071 \\
\hline Other locations & $1.066(0.879-1.292)$ & 0.517 & $1.100(0.887-1.365)$ & 0.385 & $1.209(0.920-1.589)$ & 0.172 \\
\hline \multicolumn{7}{|l|}{ Tumor grade } \\
\hline Low & 1 & & 1 & & 1 & \\
\hline High & 2.197(1.653-2.919) & $<0.001$ & $2.682(1.906-3.775)$ & $<0.001$ & $1.871(1.141-3.070)$ & 0.013 \\
\hline \multicolumn{7}{|l|}{ Tumor stage } \\
\hline Localized & 1 & & 1 & & 1 & \\
\hline Regional & $1.174(0.967-1.425)$ & 0.106 & $1.252(0.997-1.573)$ & 0.053 & $1.176(0.893-1.548)$ & 0.250 \\
\hline Distant & $2.904(2.322-3.631)$ & $<0.001$ & $3.195(2.479-4.119)$ & $<0.001$ & $2.678(1.936-3.704)$ & $<0.001$ \\
\hline \multicolumn{7}{|l|}{ Tumor size } \\
\hline$<5 \mathrm{~cm}$ & 1 & & 1 & & 1 & \\
\hline $5-10 \mathrm{~cm}$ & $1.268(0.965-1.666)$ & 0.089 & $1.280(0.939-1.744)$ & 0.118 & $1.179(0.804-1.729)$ & 0.399 \\
\hline$>10 \mathrm{~cm}$ & $1.699(1.264-2.283)$ & $<0.001$ & $1.727(1.242-2.402)$ & 0.001 & $1.778(1.161-2.724)$ & 0.008 \\
\hline \multicolumn{7}{|l|}{ Surgery } \\
\hline Yes & 1 & & 1 & & 1 & \\
\hline No & $1.604(1.333-1.931)$ & $<0.001$ & $1.460(1.194-1.785)$ & $<0.001$ & $1.405(1.080-1.829)$ & 0.011 \\
\hline \multicolumn{7}{|c|}{ Radiation treatment } \\
\hline Yes & 1 & & 1 & & 1 & \\
\hline No & $0.716(0.601-0.852)$ & $<0.001$ & $1.247(1.025-1.518)$ & 0.027 & $0.898(0.697-1.157)$ & 0.405 \\
\hline \multicolumn{7}{|l|}{ Chemotherapy } \\
\hline Yes & - & & - & & 1 & \\
\hline No & - & - & - & - & $1.337(1.066-1.678)$ & 0.012 \\
\hline
\end{tabular}

Similarly, our study revealed that tumor grade and stage were independent prognostic factors of both OS and CSS. Many studies have reported that the tumor size above $10 \mathrm{~cm}$ is associated with poorer prognosis and decreased survival rate of osteosarcoma patients [7, 24, 25]. In our cohort, tumor size $>10 \mathrm{~cm}$ was also an independent predictor of both OS and CSS.

Surgical excision and chemotherapy are considered the standard treatment strategy for osteosarcoma, but the efficacy of chemotherapy in older patients in particular is still controversial. 
Chemotherapy-related toxicity including peripheral neuropathy, hematological toxicity, and nephrotoxicity was as considerable and generally higher than those younger patients $[12,13]$. Therefore, treating the older osteosarcoma remains a challenge. Many authors hold that older osteosarcoma patients should receive aggressive chemotherapy and surgery if at all possible to achieve a survival rate similar to that observed in adolescents $[11,12,26]$. However, Iwata et al. [10] reported that definitive surgery was a significant prognostic factor, whereas chemotherapy did not influence survival, which was similar to the results of primary older osteosarcoma patients in our study. Other studies also found chemotherapy did not prolong the survival of the older osteosarcoma patients [20, 21]. However, among the secondary older osteosarcoma patients, surgical resection of primary tumors and systemic chemotherapy significantly prolonged the OS. Thus, surgery in combination with chemotherapy is recommended for the treatment of the secondary older osteosarcoma patients, while for the primary older osteosarcoma patients, only surgery is recommended.

Regarding radiotherapy, a satisfactory outcome is usually not achieved in osteosarcoma patients. Radiotherapy may offer local control as osteosarcoma is radioresistant [14]. Its impact on survival of this age group remains controversial. Some studies reported that radiotherapy was associated with the prognosis in osteosarcoma [27-29]. Schwarz et al. [28] found that the combination of surgery, chemotherapy, and radiotherapy was the optimum choice and the consistent use of full-dose chemotherapy is significant for the response to radiotherapy. However, Arshi et al. [30] found that radiation treatment was significantly associated with worse outcomes in patients with spinal osteosarcoma. Similarly, our study found that radiotherapy predicted worse OS and CSS of the primary older osteosarcoma, suggesting that radiation treatment is not an appropriate therapy for treating such patients. Among secondary older osteosarcoma patients, radiotherapy trended towards decreased survival but did not achieve statistical significance for OS on multivariate analysis. Additionally, many studies reported that radiation induced the occurrence of osteosarcoma with aggressive behaviors, suggesting that its use should be careful [31-33].

This study had several limitations. First, this study was a retrospective study from a large secondary database, which does not provide access to detailed clinical information. Prospective study should be performed to further confirm our conclusion. Second, the SEER database does not include other important information such as time to recurrence during follow-up, radiotherapy regimen and molecular pathological characteristics, which may affect the prognosis of patients. These variables may be an effective complement to this study, which will be an important section of our future research. Third, only $452(38.9 \%)$ of the primary osteosarcoma patients were older than 60 -years, whereas $289(65.1 \%)$ of the secondary osteosarcoma patients were older than 60-years. Thus, there was age difference between the primary and secondary osteosarcoma patients. Age was an independent predictor of both OS and CSS in the primary older osteosarcoma patients. However, age was not an independent predictor of OS in the secondary older osteosarcoma patients. Thus, we think the OS and CSS rates between these two groups could be compared to a certain extent. But we also think more clinical researches should be done to get more convincing results. Despite these limitations, our large sample size along with demographic and tumor data allows for the investigation of important associations and predictors of older osteosarcoma. Additionally, the SEER database provides high statistical power due to the collection of data from multiple centers.

\section{Conclusion}

We firstly and simultaneously analyzed the prognostic factors of 1162 primary older osteosarcoma patients and 444 secondary older osteosarcoma patients. Surgery in combination with chemotherapy is recommended for the treatment of the secondary older osteosarcoma patients, while for the primary older osteosarcoma patients, only surgery is recommended.

\section{Acknowledgements}

This work was supported by the grants from National Key R\&D Program of China (2018YFC1105400), National Natural Science Foundation of China (81702118), 2018 Zhejiang University Academic Award for Outstanding Doctoral Candidates and Medical and Health Science and Technology Plan of Department of Health of Zhejiang Province (WKJ-ZJ-1821).

\section{Competing Interests}

The authors have declared that no competing interest exists.

\section{References}

1. Ottaviani G, Jaffe N. The epidemiology of osteosarcoma. Cancer treatment and research. 2009; 152: 3-13.

2. Bielack SS, Kempf-Bielack B, Delling G, et al. Prognostic factors in high-grade osteosarcoma of the extremities or trunk: an analysis of 1,702 patients treated on neoadjuvant cooperative osteosarcoma study group protocols. Journal of clinical oncology. 2002; 20: 776-90. 
3. Duchman KR, Gao Y, Miller BJ. Prognostic factors for survival in patients with high-grade osteosarcoma using the Surveillance, Epidemiology, and End Results (SEER) Program database. Cancer epidemiology. 2015; 39: 593-9.

4. Mirabello L, Troisi RJ, Savage SA. Osteosarcoma incidence and survival rates from 1973 to 2004: data from the Surveillance, Epidemiology, and End Results Program. Cancer. 2009; 115: 1531-43.

5. Harrison DJ, Geller DS, Gill JD, et al. Current and future therapeutic approaches for osteosarcoma. Expert review of anticancer therapy. 2018; 18: 39-50

6. Schlegel M, Zeumer M, Prodinger PM, et al. Impact of Pathological Fractures on the Prognosis of Primary Malignant Bone Sarcoma in Children and Adults: A Single-Center Retrospective Study of 205 Patients. Oncology. 2018; 94:354-62.

7. Anderson ME. Update on Survival in Osteosarcoma. The Orthopedic clinics of North America. 2016; 47: 283-92.

8. Dray MS, Miller MV. Paget's osteosarcoma and post-radiation osteosarcoma: secondary osteosarcoma at Middlemore Hospital, New Zealand. Pathology. 2008; 40: 604-10.

9. Hamre MR, Severson RK, Chuba P, et al. Osteosarcoma as a second malignant neoplasm. Radiotherapy and oncology. 2002; 65: 153-7.

10. Iwata S, Ishii T, Kawai A, et al. Prognostic factors in elderly osteosarcoma patients: a multi-institutional retrospective study of 86 cases. Annals of surgical oncology. 2014; 21: 263-8.

11. Ozkurt B, Basarir K, Yalcin B, et al. Chemotherapy in primary osteogenic sarcoma in patients over the age of forty. Acta orthopaedica et traumatologica turcica. 2017; 51: 123-7.

12. Ferrari S, Bielack SS, Smeland S, et al. EURO-B.O.S.S.: A European study on chemotherapy in bone-sarcoma patients aged over 40: Outcome in primary high-grade osteosarcoma. Tumori. 2018; 104: 30-6.

13. Grimer RJ, Cannon SR, Taminiau AM, et al. Osteosarcoma over the age of forty. European journal of cancer. 2003; 39: 157-63.

14. Sole CV, Calvo FA, Alvarez E, et al. Adjuvant radiation therapy in resected high-grade localized skeletal osteosarcomas treated with neoadjuvant chemotherapy: Long-term outcomes. Radiotherapy and oncology. 2016; 119: $30-4$

15. Zhang W, Tanaka M, Sugimoto $Y$, et al. Carbon-ion radiotherapy of spinal osteosarcoma with long-term follow. European spine journal. 2016; 25 (Suppl 1): 113-7.

16. Ta HT, Dass CR, Choong PF, et al. Osteosarcoma treatment: state of the art. Cancer metastasis reviews. 2009; 28: 247-63.

17. Longhi A, Errani C, De Paolis M, et al. Primary bone osteosarcoma in the pediatric age: state of the art. Cancer treat reviews. 2006; 32: 423-36.

18. Worch J, Matthay KK, Neuhaus J, et al. Osteosarcoma in children 5 years of age or younger at initial diagnosis. Pediatric blood \& cancer. 2010; 55: 285-9.

19. Okada K, Hasegawa T, Nishida J, et al. Osteosarcomas after the age of 50: a clinicopathologic study of 64 cases--an experience in northern Japan. Annals of surgical oncology. 2004; 11: 998-1004.

20. Nishida $Y$, Isu K, Ueda T, et al. Osteosarcoma in the elderly over 60 years: a multicenter study by the Japanese Musculoskeletal Oncology Group. Journal of surgical oncology. 2009; 100: 48-54.

21. Carsi B, Rock MG. Primary osteosarcoma in adults older than 40 years. Clinical orthopaedics and related research. 2002: 53-61.

22. Petrilli AS, Gentil FC, Epelman S, et al. Increased survival, limb preservation, and prognostic factors for osteosarcoma. Cancer. 1991; 68: 733-7.

23. Smeland S, Muller C, Alvegard TA, et al. Scandinavian Sarcoma Group Osteosarcoma Study SSG VIII: prognostic factors for outcome and the role of replacement salvage chemotherapy for poor histological responders. European journal of cancer. 2003; 39: 488-94.

24. Colding-Rasmussen $\mathrm{T}$, Thorn AP, Horstmann $\mathrm{P}$, et al. Survival and prognostic factors at time of diagnosis in high-grade appendicular osteosarcoma: a 21 year single institution evaluation from east Denmark. Acta oncologica. 2018; 57: 420-5.

25. Parry MC, Laitinen M, Albergo J, et al. Osteosarcoma of the pelvis. The bone \& joint journal. 2016; 98-b: 555-63.

26. Bacci $G$, Ferrari $S$, Donati $D$, et al. Neoadjuvant chemotherapy for osteosarcoma of the extremity in patients in the fourth and fifth decade of life. Oncology reports. 1998; 5: 1259-63.

27. Matsunobu A, Imai R, Kamada T, et al. Impact of carbon ion radiotherapy for unresectable osteosarcoma of the trunk. Cancer. 2012; 118: 4555-63.

28. Schwarz R, Bruland O, Cassoni A, et al. The role of radiotherapy in oseosarcoma. Cancer treatment and research. 2009; 152: 147-64.

29. Matsumoto K, Imai R, Kamada T, et al. Impact of carbon ion radiotherapy for primary spinal sarcoma. Cancer. 2013; 119: 3496-503.

30. Arshi A, Sharim J, Park DY, et al. Prognostic determinants and treatment outcomes analysis of osteosarcoma and Ewing sarcoma of the spine. The spine journal. 2017; 17: 645-55.

31. Liao LQ, Yan HH, Mai JH, et al. Radiation-induced osteosarcoma of the maxilla and mandible after radiotherapy for nasopharyngeal carcinoma. Chinese journal of cancer. 2016; 35: 89.

32. Puhaindran ME, Hamilton $\mathrm{K}$, Schlumbohm $\mathrm{S}$, et al. Radiation-induced osteosarcoma of the hand: case report. The Journal of hand surgery. 2014; 39: $1151-4$.

33. Rashidghamat E, Calonje JE. A case of radiation-induced osteosarcoma of the skull presenting as a cutaneous epidermotropic tumor with a short latent period. Journal of cutaneous pathology. 2018; 45: 352-4. 